\title{
An Energy Efficient Critical Event Monitoring Routing Method for Wireless Sensor Networks
}

\author{
Yogesh Y. Shinde. \\ Department Of Electronics and Telecommunication \\ Dr. D. Y. Patil S. O. E. Lohegaon, \\ Pune, Maharashtra India
}

\author{
Santosh S. Sonavane, Ph.D \\ Department Of Electronics and Telecommunication \\ Dr. D. Y. Patil S. O. E. Lohegaon, \\ Pune, Maharashtra India.
}

\begin{abstract}
In wireless sensor network (WSN), due to restraint of node energy, energy efficiency is an important factor that should be considered when designing the protocol. In order to save energy data combination and aggregation should be exploited in this case decreasing energy consumption and communication cost by aggregate redundant data at transitional node which reducing size and number of exchange messages. The network formed is ad-hoc network, as nodes in this network communicate with each other without any infrastructure. This paper proposed a new improved algorithm EEDRINA was compared to two other known solutions LowEnergy Adaptive Clustering Hierarchy (LEACH) and Data Routing for In -Network Aggregation (DRINA) algorithms which is intended to improve the routing technique to improve routing performance as well as balance energy consumption of the entire network and extend the life of network in critical event monitoring. This work is emulated by NS2 platform, the simulation results indicates that our proposed EEDRINA solution outperforms these LEACH \& DRINA known solutions in different scenarios and in different parameters required by WSN.
\end{abstract}

\section{Keywords}

Broadcasting, Critical event monitoring, Lifetime of nodes, routing protocol, Wireless Sensor Network (WSN)

\section{INTRODUCTION}

Wireless sensor network (WSN) consist of a group of spatially distributed and devoted sensors for monitoring and recording the physical conditions of the environment and organizing the collected data at a central location. WSNs measure environmental conditions like, humidity, temperature, pollution level, sound, and direction, pressure, etc [27]. There are large no of nodes in target area. Nodes in target area (WSN) are communicated with each other for a given task. Nodes or devices of WSN are powered by batteries this is the projecting feature of WSN. Energy saving is most important issue in WSN, as Sensor nodes have to work for a long time (couple of years) without recharging their batteries. A tiny packet wants to be transmitted in critical event monitoring. The alarm packet needs to be broadcast to the entire network hence broadcast delay is an important issue in critical event monitoring.

Sleep scheduling and different routing methods are constantly used for event monitoring as WSN are likely to operate for a long time (in some cases many years) devoid of recharging their batteries. Transmission delay should introduced in sleep scheduling for the cause that node should wait until receiver nodes are active and prepared to accept the message. Wireless Sensor Networks are frequently produce a large amount of information that wants to be routed towards sink node in multihop fashion also known as Data Driven Networks. Hence in data gathering process routing plays an important role.
Delivery of sensed data even in presence of interruption in communication and nodes failures is one of the main challenges in routing algorithms for WSN. When data aggregation is performed along the routing path this failures become more critical as packet with aggregated data contains information from various sources. Considerable amount of information will be lost whenever one of these packets lost. High aggregation rate, maximize number of overlapping routes, reduced number of messages for creation a routing tree and also reliable data transmission these are desirable characteristics of data aggregation routing protocols in the context of WSN. In order to overcome these challenges, in this work, we propose a novel Energy Efficient Data Routing for In-Network Aggregation (EEDRINA) for WSN.

\section{LITERATURE SURVEY}

To reduce ideal listening energy cost Wei Ye et. al [1] introduces The S-MAC medium access protocol in which sensor nodes synchronized by periodic duty cycle. In S-MAC each node synchronized with its neighboring nodes and follows a periodic sleep/active schedule. During active period radios are on to transmit and receive messages then they turn back to sleep periods in these periods the radios are completely turn off. Although S-MAC is energy efficient, it has one main drawback it introduces sleep latency. At a transmitting node or source node, if a sampling reading occur during the sleep period it has to be wait for the active period come. An intermediate node has to wait to forward packet which comes from previous hope to destination or receiver until receiver wake up this delay is called as sleep latency

Time division multiple access (TDMA) condition based protocols set up fixed time periods for nodes to communicate hence it eliminate the channel conflict and idle listening energy costs. The Wendi Rabiner Heinzelman et. al [2] and Guangyu Pei et. al introduced Low-Energy Adaptive Clustering Hierarchy (LEACH) and Power-Aware Clustered TDMA (PACT) respectively these techniques offers Centralized cluster management which has ability for a single node to manage traffic exchanges. Protocols make use of an "off-line" self-selection technique. These protocols spend considerable energy in exchanging control packets to move vacant time slots or require complex algorithms to allocate time slots based upon previous traffic requirements, to use bandwidth efficiency.

Abtin Keshavarzian et. al [4] presented multi-parent schemes, in which cross-layer approach where multiple routes for transfer of messages and wakeup schedules for various nodes are crafted in synergy to increase longevity while reducing message delivery latencies. N.A. Vasanthi and Sannadurai [5] defined a new Sleep schedule (Q-MAC) for Query based sensor networks that give energy efficient data transmission with minimum end-to-end latency. Using the static schedule the radios of the nodes sleep extra whenever there is no query. The sleep schedule is changed dynamically with respect to 
query, In Q-MAC the energy is minimized up to $80 \%$ as compare to S-MAC with minimum energy consumption

The related data according to the query is collected by the network and flow the results to the user in a period, in querybased approach. If the transmitting data out of the network is raw in real time is also energy expensive. There are a number of query processing techniques like Tiny DB, COUGAR and ACQUIRE. Y. Yao et.al [6] and Narayanan Sadagopan et.al [7] proposed network aggregation for data organization with compact energy consumption. In Tiny DB each sensor for every sample period is 'on' for exactly the same four seconds. In COUGAR network is activated exactly once per period this gives significant energy saving but it increases delay. ACQUIRE outcome in significant energy savings but it gives higher latency.

N. A. Vasanthi and S. Annadurai [8] explained Asynchronous Wakeup Schedule (AWS) algorithm for the radio of the nodes in wireless sensor networks in which each node is assigned a specific color. Wake up schedule of node's radio is set, derived from the color assigned to that particular node. Each node has a forwarding table, gives the color information of the neighboring nodes. Active neighbor detection is done using the forwarding table, when a data packet has to be transmitted. Which helps to discover out the neighboring node that becomes active earlier gives least waiting time for packet helps minimizes the overall end to end delay.

Scott C. et.al [9] proposed Minimum-Latency Broadcast Scheduling (MLBS) method in wireless ad hoc networks represented by unit-disk graphs, $\mathrm{R}$ is the maximum distance of all the nodes from the source of the broadcast. The previously algorithm for MLBS in UDGs, Basic Broadcast Schedule (BBS), Pipelined Broadcast Schedule (PBS) Enhanced Broadcast Schedule (EBS).They create a broadcast schedule with latency 648R. Three progressively improved approximation algorithms for MLBS present in this paper. They create broadcast schedules with latency at $24 \mathrm{R}-23, \mathrm{R}+$ $\mathrm{O}(\log \mathrm{R})$ and $16 \mathrm{R}-15$ respectively.

N. Bouabdallah et. al [10] focuses on the class of CM applications where the end user has to send the most recent event to the central node or sink node here each node produces information Hence sensor node continuously transmit their information ,regardless of whether they have relevant data. In CM-EDR system an event is defined as an important change in the supervised incident compared to the last reading sent to the sink node.

In ADB presented by Yanjun Sun et.al [11] the progress of the broadcast is optimizes at the level of transmission from the node to all neighbor independently, due to this it avoids unnecessary transmissions, allows next hops to quickly initiate forwarding the broadcast, and enables nodes to go to sleep again as soon as possible. Like this, ADB achieves high energy efficiency, near optimal latency, and high packet delivery radio. ADB is therefore efficient in distributing small messages when asynchronous duty-cycling is used, for services such as resource discovery and routing.

Ping Song et.al [12] represented Physical layer integrated synchronization protocol (PLISP) is a new time synchronization protocol. Physical layer integrated synchronization protocol adds timestamp in physical layer to decrease uncertainty of message exchange process. Results gives that for single hop time synchronization requires $1 \mu$ s but its consumption is higher than TPSN (Timing sync protocol for sensor networks) system
Neighbor aware cluster head protocol defined by Tarek $\mathrm{R}$. Sheltami et. al [13] divides network into clusters and uses cluster based communications. Cluster head serve each group of nodes. NAC uses (LINT) Local Information No Topology and (LILT) Local Information Link State Topology protocols to optimized number of neighbors served by cluster head also (RBS) algorithm to allow the nodes to go to sleep mode and synchronized with their cluster head. The results conclude that if the application has delay constraint this protocol gives good results as compare to SMAC and DMAC protocols.

A Boukerche et.al [14] J. Al-Karaki et.al [15] and E. Fasolo[16] proposed protocols are usually based on Tree Based Approach in which some special nodes are elected to work as aggregation points and define preferred direction to be followed to forward aggregate data from source to sink node. In these protocols first construct tree structure of nodes and then used afterward to either route the data or react to queries sent by sink node. When two or more packets arrive at same node aggregation of data is performed during routing. On the other hand this approach has some drawbacks. For example data from whole sub tree will be lost when packet is lost at certain level of tree due to channel impairments.

Cluster Based Approaches presented in E. F. Nakamura et al [17], A. P. Chandrakasan [18] and Leandro Aparecido Villas et. al [19] also consist of hierarchical organization of the network but in these approaches, nodes are divided into clusters. In addition cluster heads are the special nodes are elected to aggregate data locally and forward the result to the sink node

A. P. Chandrakasan et. al [18] introduced LEACH (LowEnergy Adaptive Clustering Hierarchy) algorithm in which cluster head acts as aggregation points and communicate directly to the sink node. Cluster heads are randomly chosen to in each round with the intention of evenly distribute energy consumption among all nodes. This algorithm assumes that sink can be reached by any node within one hope, which limits the size of network.

For each event InFRA (The Information Fusion-based Role Assignments) algorithm presented by E. F. Nakamura et. al [17] builds a cluster with those nodes that were able to detect it. The cluster head aggregate the data contained by the cluster and send the results towards the sink node. The aim of InFRA algorithm is to build the short path tree that maximizes information synthesis. A disadvantage of InFRA algorithm is that the information of new event that arises in the network must be flooded throughout the network to update the aggregated coordinator distance and to inform other nodes about its occurrence due to this communication cost of the algorithm increases, thus, limits its scalability.

Laendro Aparecido Villas et. al [19] proposed cluster based DRINA algorithm. This algorithm creates a cluster of those nodes that detects a critical event as well as election of cluster head. DRINA routing protocol tends to use fewer control packets and maximize the aggregation points to build the routing tree. DRINA does not flood message to whole network whenever new event occurs. This is main difference between DRINA and InFRA

\section{INTRODUCTION TO LEACH PROTOCOL}

Cluster is formed dynamically, which is small group of nodes. All nodes in sensor network allowed contenting for a position of a cluster head and then finally selecting suitable cluster head. In this protocol there are two phases 


\subsection{Clustering Phase}

LEACH Protocol [20][21] is representative of routing protocol. It is self organized and self adaptive. It uses round as a unit. A node randomly picks a number between 0 to 1 , compare this randomly selected number to the $\mathrm{T}(\mathrm{n})$ which is a threshold value. If the randomly selected number is less than $T(n)$ in current round rotation then it become cluster head in respective round, else it become common node. $\mathrm{T}(\mathrm{n})$ could be define as follows[22]

$$
\begin{gathered}
T(n)=\left[\frac{P}{1-\mathrm{P}\left(r * \bmod \frac{1}{P}\right)}\right], \quad \text { if } n € G \\
T(n)=0 \text { otherwise }
\end{gathered}
$$

Where ' $\mathrm{n}$ ' represent current node, 'P' represents desired percentage of clusterhead in all sensor population. Current round number is represented by ' $r$ '. ' $G$ ' is a set of nodes that are not selected as a cluster head in their last $1 / p$ round

\subsection{Data Transfer Phase}

In this phase nodes in respective clusters send their data to clusterhead. Clusterhead would combine data and send it to sink node. Data transfer phase is longer than clustering phase.

\section{INTRODUCTION TO DRINA PROTOCOL}

The main goal of DRINA protocol [19] is maximize data aggregation and create a routing tree with shortest path that connect all source node to the sink. Nodes are divided into following four categories in the routing infrastructure creation:

- Collaborator. A node that detects an event

- Coordinator. A node that also detects an events. It gathers data sent by collaborator node, aggregating them and sends the result to the sink node.

- Sink. A node that receives data from set of coordinator and collaborator

- Relay. A node that forwards that data towards sink

The DRINA algorithm is divided into three phases 1. Building the Hop Tree 2. Cluster pattern 3. Routing Formation and Hop Tree Update.

\subsection{Building the Hop Tree}

In this phase [19] distance of each node from sink is measured in terms of hops. This phase is start with sending Hop Configuration Message from sink node to all network nodes. The HCM message contains two fields: ID and Hop To Tree, where ID is identifier of node which started or retransmitted HCM message and Hop To Tree is distance measured in hops, by which an HCM message is passed.

Algorithm 1. Hop to Tree Configuration Phase

Node sink sends a broadcast of HCM message with the value of Hop To Tree $=1$

// $R_{U}$ is the set of nodes that the message HCM//

For each $u$ out of $R_{U}$ do

If Hop To Tree $(\mathrm{u})>$ Hop To Tree (HCM) and

First sending $(u)$ then
Next hop $=\mathrm{ID}_{\mathrm{HCM}}$;

Hop To Tree $_{\mathrm{U}}=$ Hop To Tree $\mathrm{HCM}^{+1}$;

$\mathrm{HCM} / /$

//Node u update the value of ID field in the message

$\mathrm{ID}_{\mathrm{HCM}=} \mathrm{ID}_{\mathrm{u}}$;

// Node u update the value of the HOP To Tree field in message $\mathrm{HCM} / /$

Hop To Tree HCM $=$ Hop To $_{\text {Tree }}$;

// Node $\mathrm{u}$ sends a broadcast message of HCM with the new values//

First Sending $\mathrm{u}_{\mathrm{u}}=$ False;

end

else

Node u discards the receive message HCM;

end

end

\subsection{Cluster Formation}

When one or more nodes detect an event, the leader selection algorithm [19] starts and sensing nodes will be in a row for headship. Only one node will be declared as a leader (coordinator) at the end of this selection algorithm.

Algorithm 2. Cluster Formation and Leader Election

Input: S // Set of nodes that detected the events//

Output: $\mathrm{u} / / \mathrm{A}$ node of set $\mathrm{S}$ is elected leader of the group//

For each $\mathrm{u}$ out of $\mathrm{S}$ do

roleu =coordinator;

// node u sends message MCC in broadcast//

Announcement of the event detection;

$/ / \mathrm{Nu}$ is the set of neighbors of node $\mathrm{u}$

For each w out of Nu do

If Hop To Tree (u)> Hop To Tree (w) then

Roleu = collaborator;

Node $\mathrm{u}$ retransmits the MCC message received from node w;

end

else if Hop To Tree $(\mathrm{u})=$ Hop To Tree $(w)$ and ID $(\mathrm{u})$ $>\mathrm{ID}(\mathrm{w})$ then

Roleu = collaborator; node w;

Node retransmits the MCC message received from

end

else

Node u discards the MCC message received from w;

end

end 


\subsection{Routing Formation and Hop Tree Update}

The selected group leader establishes the new route for the event broadcasting. This process is mention in algorithm 3[19]. The resulting route is tree that connects coordinator nodes to sink after this hop tree updating begins the main goal of this phase is to update Hop to Tree of each node from newly established route. This hope updating follows the same principle described in algorithm 1

Algorithm 3. Route establishment and hope tree update

Leader node $\mathrm{V}$ of new event sends a message REM to its Next HopV

Repeat

$/ / \mathrm{u}$ is node that receives the REM message, that was sent by node $\mathrm{V} / /$

If $\mathrm{u}=\mathrm{Next}$ hopeV then

Hop To Tree $\mathrm{U}=0$;

// node $\mathrm{u}$ is the part of new route built//

Roleu = Relay;

Node U sends the message REM to its Next Hopu;

Node $\mathrm{u}$ broadcast the massage HCM with the value of Hop

To Tree $=1$;

Node that receives the message sent by node $\mathrm{u}$, will run command line 2 until line 14 of algorithm 1;

End

Until find out sink node or a node belonging to the routing structure already established;

Repeat

$$
\begin{aligned}
& \text { // sonsu is the number of decrement of } \mathrm{u} / / \\
& \text { If sonsu }>1 \text { then } \\
& \text { Aggregate all the data and sends it to the }
\end{aligned}
$$

Next hop u;

$$
\text { If Roleu=Relay then }
$$

Execute mechanism of route repair mechanism

$$
\text { End }
$$

End

else

send data to Next hop u;

If Roleu=Relay then

Execute mechanism of route repair

mechanism

End

$$
\text { End }
$$

Until the node has data to transmit/receive

\section{EEDRINA PROTOCOL}

This is extension of DRINA protocol hence it uses all existing algorithms of DRINA as well as new algorithm given below for energy performance improvement.

The Energy aware routing plays an important role for moderately allocates the traffic load among all the participating nodes in the network. For example consider a fig. 2 (a) the path A-B-C-D is the optimal path connecting from A to D. We measure hop count for shortest path routing. Thus, nodes B and $\mathrm{C}$ will continuously engage in forwarding the traffic, thus other nodes are free from the traffic. As a result residual energy of nodes becomes extensively varied. If the routing is not energy aware nodes $\mathrm{B}$ and $\mathrm{C}$ die early due to drain out of battery supply. Here we proposed the EEDRINA algorithm based on route redirection. The route will gradually meet to an option node disjoint path with this successive local redirection operation.

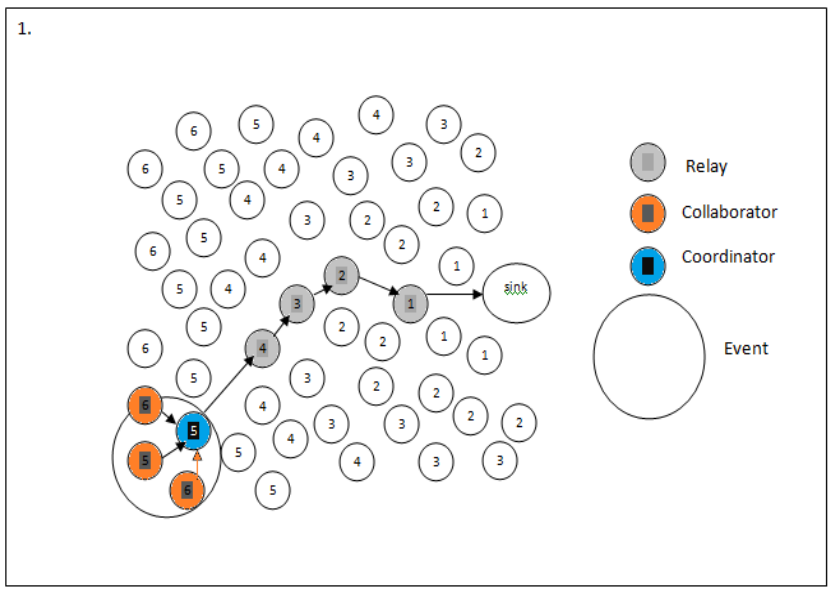

(a) Example of routing tree to 1 event

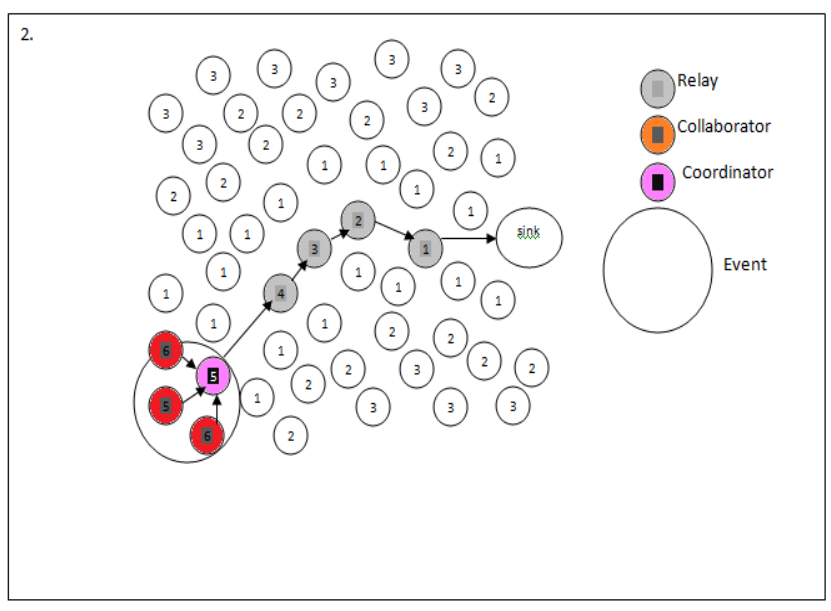

(b) Update of Hop Tree

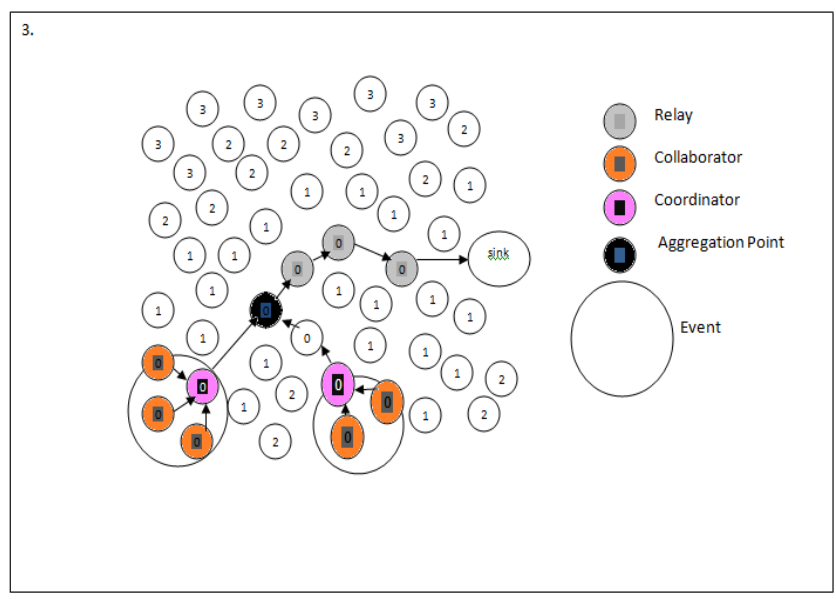

(c) Example of the routing tree to event

Fig. 1. Example of establishing new routes and updating the hop tree

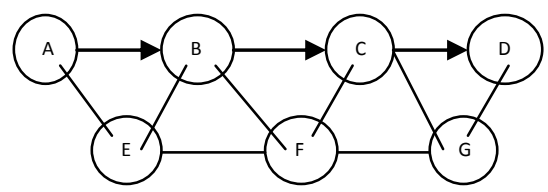

a) primary Path that drains the residual energy at nodes $B$ and $C$ 


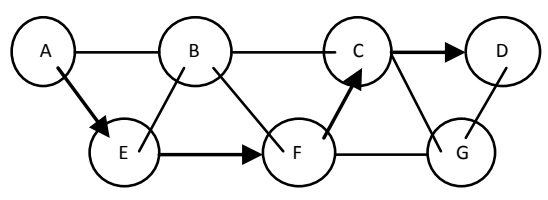

b) Node B circumvented after route redirection

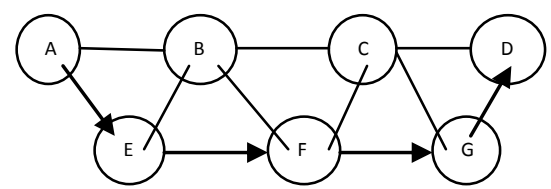

c) Node $\mathbf{C}$ circumvented after route redirection. B node disjoint new path is found

Fig.2: Example of successive local route redirection operations. (The links shown in fig. are wireless)

Here we proposed the EEDRINA algorithm based on route redirection. The route will gradually meet to an option node disjoint path with this successive local redirection operation.

Figure 3 shows two basic redirections. In case 1, fig 3 (a), we consider that three successive nodes $\mathrm{P}, \mathrm{Q}$ and $\mathrm{R}$ on the path on A-D connection. Node $S$ is common neighbor node of all three nodes. Sequentially transmitted data packets by three nodes $(\mathrm{P}$, $\mathrm{Q}$ and $\mathrm{R}$ ) can easily overhear by node $\mathrm{S}$. Here node $\mathrm{S}$ can overhear the same packet three times. By identifying this scenario node $S$ realize that it can replace node $Q$, here, the sub-path P-Q-R can be redirected to P-S-R. If node S sees that power level of $S$ is more than that of $Q$ and the difference of energy level is significant enough, node $S$ will do the redirection

As shown in Fig. 3(b), we consider that difference in the energy level between $\mathrm{T}$ and $\mathrm{V}$ is considerable adequate. The same is true between $\mathrm{W}$ and $\mathrm{T}$. When the data packets travel along $\mathrm{S}, \mathrm{T}$ and $\mathrm{R}$ nodes, node $\mathrm{V}$ will observe that node $\mathrm{T}$ desires to be circumvented.

Node $\mathrm{V}$ also knows that it is a neighbor of both node $\mathrm{T}$ and node $\mathrm{S}$ which is its up-stream route neighbor. Similarly, node $\mathrm{W}$ will observe that node $\mathrm{T}$ needs to be circumvented and node $\mathrm{W}$ is neighboring with node $\mathrm{T}$ which is its down-stream root neighbor and node R. Even from locally overheard information, nodes $\mathrm{V}$ and $\mathrm{W}$ can differentiate their different role. It is up to node $\mathrm{W}$ to find out continuation of node $\mathrm{V}$. To make easy this process, $\mathrm{W}$ broadcast the message saying that "Node T needs help, and I am its down-stream helper, who is its up-stream helper?" If node $\mathrm{V}$ replies this message with acknowledgement here wireless link between $\mathrm{V}$ and $\mathrm{Q}$ is identified. The two helper nodes will do the redirection to replace S-T-R sub-path by the new S-V-W-R-path.

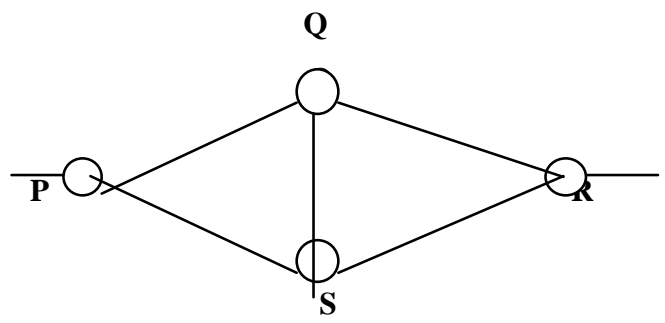

a) 2 step alternate sub-path, P-S-R

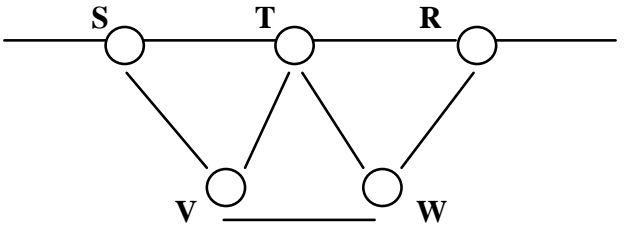

b) 3 step alternate sub-path, $S-V-W-R$

Fig.3: Basic alternative sub-paths cases. (The links shown in fig. are wireless)

Consider an example shown in figure 2(a), nodes B and C both have low energy level. After one redirection as shown in fig. 2 (b) path A-B-C-D becomes A-E-F-C-D here node B is circumvented. Then node $\mathrm{C}$ is circumvented and path becomes A-E-F-G-D as shown in fig. 2 (c).

\subsection{EEDRINA Algorithm}

Two more fields we need to add to the conventional IP-header of each packet. They are "Residual Energy level "and "Hop Counter". We use REL (P) and HC(P) respectively to represent these two additional fields on the packet. The other fields in the packet needs to access in this algorithm such as sender, sequence number, source and destination, they are represented by $\operatorname{SEN}(\mathrm{P}), \operatorname{SEQ}(\mathrm{P}), \operatorname{SRC}(\mathrm{P})$ and $\operatorname{DEST}(\mathrm{P})$ respectively. We use SRC-DEST $(\mathrm{P})$ to symbolize the source-destination pair of the flow that the packet belongs to. Each node maintain an "overhear table". The table contains the following three fields in each entry: sequence number, source-destination pair and "overhear list".

Algorithm: When packet $\mathrm{P}$ is overhear by node $\mathrm{i}$,

BEGIN

Step 1: Find SRC-DEST $(\mathrm{P})$ in overhear table;

Step 2: Add entry e', if no match: SRC-DEST(e')=SRC-

$\operatorname{DEST}(\mathrm{P}), \mathrm{SEQ}\left(\mathrm{e}^{\prime}\right)=\mathrm{SEQ}(\mathrm{P}), \mathrm{O}-$ list $\left(\mathrm{e}^{\prime}\right)$ initialized with first entry $\langle\mathrm{HC}(\mathrm{P}), \operatorname{REL}(\mathrm{P}), \mathrm{SEN}(\mathrm{P})\rangle$. GOTO END;

Step 3: (Suppose a match is found at entry e.) Ignore $P$, if SEQ(P) $<$ SEQ(e). GOTO END;

Step 4: If $S E Q(P)>S E Q(e)$, update e as the following:

$\mathrm{SEQ}(\mathrm{e})=\mathrm{SEQ}(\mathrm{P}), \mathrm{O}-$ list $(\mathrm{e})$ reset as having only one entry $\langle\mathrm{HC}(\mathrm{P}), \mathrm{REL}(\mathrm{P}), \mathrm{SEN}(\mathrm{P})\rangle$. GOTO END;

Step 5: If $\operatorname{SEQ}(\mathrm{P})==\mathrm{SEQ}(\mathrm{e})$, do the following:

Step 5.1: Add entry $\langle$ HC(P), REL(P),SEN(P)> into O-list(e);

Step 5.2: If O-list (e) has three entries $\mathrm{P}, \mathrm{Q}, \mathrm{R}$ fulfilling the following conditions, a better sub-path is found.

1) $\mathrm{HC}(\mathrm{R})==\mathrm{HC}(\mathrm{Q})+1==\mathrm{HC}(\mathrm{P})+2$;

2)REL(node i) $\geq \operatorname{MAX}(\operatorname{REL}(\mathrm{P}), \operatorname{REL}(\mathrm{R})$ );

3) (REL(node i)-REL(Q)) $\geq 2$. Stimulate this new sub-path

Drop entry e from overhear table. GOTO END;

Step 5.3: If O-list(e) has two entries $P$ and $Q$, such that $\mathrm{HC}(\mathrm{Q})==\mathrm{HC}(\mathrm{P})+1$ and $\mathrm{REL}($ node $\mathrm{i}) \geq$

$\operatorname{MAX}(\operatorname{REL}(\mathrm{P}), \operatorname{REL}(\mathrm{Q})+2)$, add this indicator I in the Waiting Indicator list: candidate $(\mathrm{I})=\mathrm{Q}, \mathrm{SEQ}(\mathrm{I})=\mathrm{SEQ}(\mathrm{e}), \mathrm{SRC}$ $\operatorname{DEST}(\mathrm{I})=$ SRC-DEST(e). GOTO END;

Step 5.4: If O-list(e) has two entries $Q$ and $R$, such that $\mathrm{HC}(\mathrm{R})==\mathrm{HC}(\mathrm{Q})+1$ and $\mathrm{REL}($ node $\mathrm{i}) \leq$

$\operatorname{MAX}(\operatorname{REL}(\mathrm{Q})+2, \operatorname{REL}(\mathrm{R}))$, node i broadcast one DRINA informing packet $\mathrm{P}^{\prime}$ as follows: candidate $\left(\mathrm{P}^{\prime}\right)=\mathrm{Q}$, $\mathrm{SEQ}\left(\mathrm{P}^{\prime}\right)=\mathrm{SEQ}(\mathrm{e}) \mathrm{SRC}-\mathrm{DEST}\left(\mathrm{P}^{\prime}\right)=\mathrm{SRC}-\mathrm{DEST}(\mathrm{e})$;

\section{PERFORMANCE EVALUATION}

In this section, we estimate the proposed EEDRINA algorithm and compare its performance to LEACH and DRINA known routing protocols. We evaluate the EEDRINA algorithm performance under the following parameters: 
1. Average energy consumption

2. Average Throughput

3. Packet Delivery Ratio

4. Average End to End Delay

5. Normalized Routing Load

Table1.Simulation Environment Parameter

\begin{tabular}{|c|c|}
\hline Environment Variable & Associated Values \\
\hline Simulation tool & Ns-allinone-2.32 \\
\hline Channel used & Wireless \\
\hline Initial Energy & Omni Directional \\
\hline Antenna Type & IEEE $802-11$ \\
\hline MAC protocol & $100,150,200,250,300$ \\
\hline Total no. of nodes & 50 \\
\hline Queue length & Two Ray Ground Model \\
\hline Propagation Model & $100 \mathrm{~s}$ \\
\hline Simulation Time & \\
\hline
\end{tabular}

\subsection{Average Energy Consumption}

Energy is one of the biggest constraints for a WSN. In general, energy consumption in sensor nodes can be divided into three parts: (i) energy consumption by sensing unit, (ii) energy consumption for communication among sensor nodes, and (iii) energy consumption due to computation.

Table 2. Average Energy Consumption (joules)

\begin{tabular}{|c|c|c|c|c|c|c|}
\hline $\begin{array}{c}\text { Sr. } \\
\text { No }\end{array}$ & Algorithm & $\begin{array}{c}100 \\
\text { nodes }\end{array}$ & $\begin{array}{c}150 \\
\text { nodes }\end{array}$ & $\begin{array}{c}200 \\
\text { nodes }\end{array}$ & $\begin{array}{c}250 \\
\text { nodes }\end{array}$ & $\begin{array}{c}300 \\
\text { nodes }\end{array}$ \\
\hline 1 & LEACH & 7.3163 & 8.5232 & 7.4858 & 7.2893 & 8.7373 \\
\hline 2 & DRINA & 7.3069 & 8.0468 & 7.4727 & 7.2737 & 8.7247 \\
\hline 3 & EEDRINA & 6.5762 & 7.2421 & 6.7345 & 6.5474 & 7.8523 \\
\hline
\end{tabular}

\section{Average Energy Consumption [Jules]}

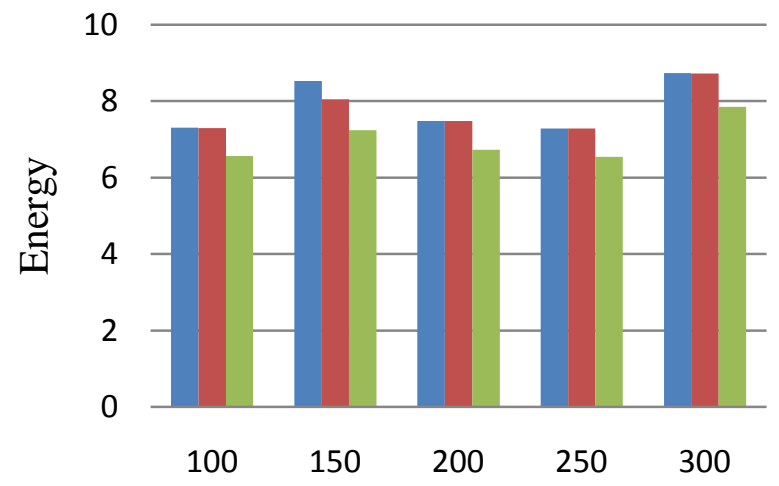

Varying Number of Sensors

- LEACH DRINA EEDRINA

Fig. 4. Average Energy Consumption
As we know that energy of the WSN is restricted, so energy consumption is important factor to evaluate the performance of it. From fig. 4 is the Average energy consumption graph, EEDRINA protocol reduced the energy consumption. This protocol should save about 10 percent energy than DRINA.

\subsection{Average Throughput}

The throughput [23] is defined as the total amount of data a receiver actually receives from the sender divided by the time between receiving the fast packet and last packet. The Average Throughput is the throughput per unit of time. . The Average Throughput is the throughput per unit of time. The throughput [23] is defined as the total amount of data a receiver actually receives from the sender divided by the time between receiving the fast packet and last packet. The Average Throughput is the throughput per unit of time.

Table 3. Average throughput

\begin{tabular}{|c|c|c|c|c|c|c|}
\hline $\begin{array}{c}\text { Sr. } \\
\text { No }\end{array}$ & Algorithm & $\begin{array}{c}100 \\
\text { nodes }\end{array}$ & $\begin{array}{c}150 \\
\text { nodes }\end{array}$ & $\begin{array}{c}200 \\
\text { nodes }\end{array}$ & $\begin{array}{c}250 \\
\text { nodes }\end{array}$ & $\begin{array}{c}300 \\
\text { nodes }\end{array}$ \\
\hline 1 & LEACH & 26.84 & 22.60 & 23.54 & 23.70 & 23.83 \\
\hline 2 & DRINA & 26.86 & 23.71 & 23.66 & 23.70 & 23.99 \\
\hline 3 & EEDRINA & 33.58 & 29.64 & 29.58 & 29.63 & 29.99 \\
\hline
\end{tabular}

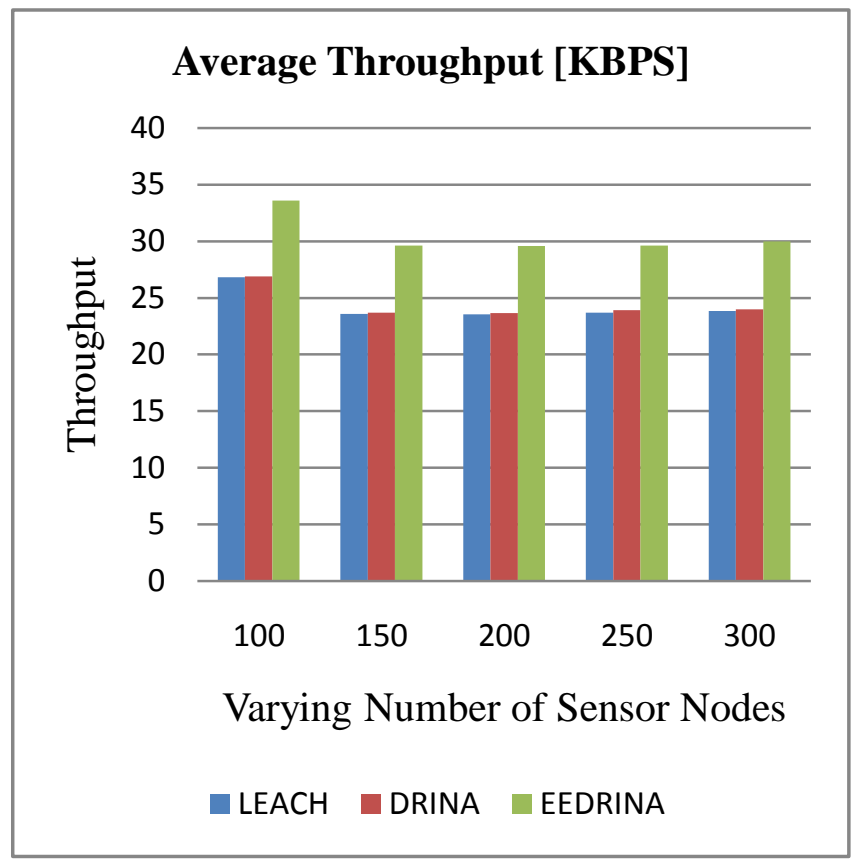

Fig. 5. Average Throughput

Fig.5. Shows average throughput of network of various numbers of nodes. Proposed EEDRINA protocol gives 25 percent more throughput than DRINA and LEACH methods which are necessary for proper communication.

\subsection{Packet Delivery Ratio}

Packet Delivery Ratio (PDR) is the ratio of the successfully delivered packets to those generated by CBR sources. The higher value of the PDR, the lesser the packet loss rate, the more efficient the routing protocol from the data delivery point of view. This number presents the effectiveness of a protocol Packet Delivery Ratio $=$ Received Packet/Send Packet $* 100$. Higher value implies better performance [24].To established proper communication Packet Delivery ratio is an important 
parameter from network point of view between source and destination. As shown in fig. 6 with proposed method it is high as compared to existing methods.

Table 4. Packet Delivery Ratio

\begin{tabular}{|c|c|c|c|c|c|c|}
\hline $\begin{array}{c}\text { Sr. } \\
\text { No }\end{array}$ & Algorithm & $\begin{array}{c}100 \\
\text { nodes }\end{array}$ & $\begin{array}{c}150 \\
\text { nodes }\end{array}$ & $\begin{array}{c}200 \\
\text { nodes }\end{array}$ & $\begin{array}{c}250 \\
\text { nodes }\end{array}$ & $\begin{array}{c}300 \\
\text { nodes }\end{array}$ \\
\hline 1 & LEACH & $99.8 \%$ & $98.4 \%$ & $98.9 \%$ & $99.6 \%$ & $99.2 \%$ \\
\hline 2 & DRINA & 100 & $99.1 \%$ & $99.2 \%$ & $99.7 \%$ & $99.5 \%$ \\
\hline 3 & EEDRINA & 100 & $99.6 \%$ & $99.3 \%$ & $99.7 \%$ & $99.5 \%$ \\
\hline
\end{tabular}

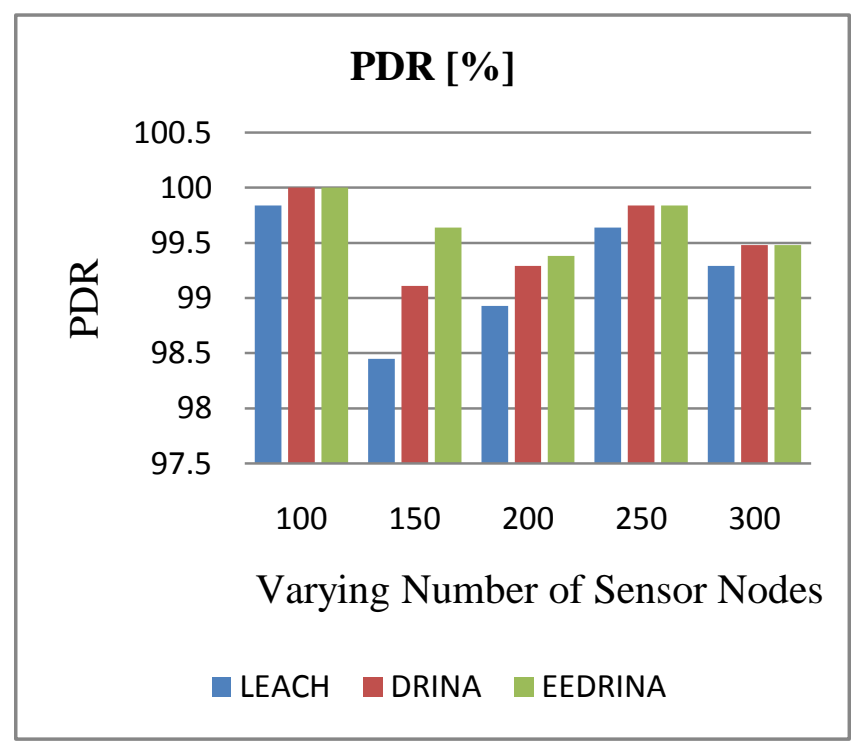

Fig. 6. Packet Delivery Ratio

\subsection{Average End To End Delay}

End-to-end delay [25] indicates how long time it took for a packet to travel from the source to the receiver. The end-to-end delay calculates the delay of the packet which is successfully transmitted from the source to the destination. This end-to-end delay includes all possible delays caused by buffering during route discovery latency, queuing in the interface queue, retransmission delays at the MAC, propagation and transfer times [26]. In WSN End to End Delay should be minimum in between source and Destination. As shown in fig .7 It is minimum than existing protocols for different nodes scenarios.

\subsection{Normalized Routing Load}

Normalized Routing load is ratio of number of routing packets to data packets. For routing protocol the value of routing load should be less.

As shown in fig.8. Routing load of EEDRINA gives 5 to 10 percent minimum value as compare to DRINA and LEACH protocols.

Table 5. Average End to End Delay (Sec.)

\begin{tabular}{|c|c|c|c|c|c|c|}
\hline $\begin{array}{c}\text { Sr. } \\
\text { No }\end{array}$ & Algorithm & $\begin{array}{c}100 \\
\text { nodes }\end{array}$ & $\begin{array}{c}150 \\
\text { nodes }\end{array}$ & $\begin{array}{c}200 \\
\text { nodes }\end{array}$ & $\begin{array}{c}250 \\
\text { nodes }\end{array}$ & $\begin{array}{c}300 \\
\text { nodes }\end{array}$ \\
\hline 1 & LEACH & 0.0583 & 0.1484 & 0.0365 & 0.0667 & 0.0558 \\
\hline 2 & DRINA & 0.0572 & 0.1480 & 0.0364 & 0.0665 & 0.0547 \\
\hline 3 & EEDRINA & 0.0570 & 0.1478 & 0.0363 & 0.0665 & 0.0545 \\
\hline
\end{tabular}

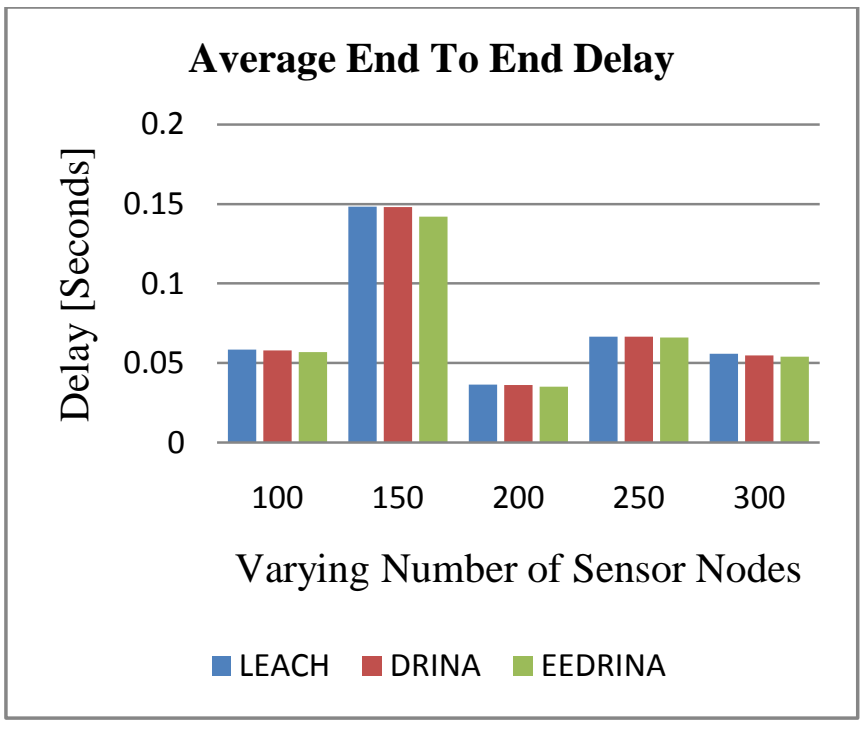

Fig. 7. Average End To End Delay

Table 6. Normalized Routing Load

\begin{tabular}{|c|c|c|c|c|c|c|}
\hline $\begin{array}{c}\text { Sr. } \\
\text { No }\end{array}$ & Algorithm & $\begin{array}{c}100 \\
\text { nodes }\end{array}$ & $\begin{array}{c}150 \\
\text { nodes }\end{array}$ & $\begin{array}{c}200 \\
\text { nodes }\end{array}$ & $\begin{array}{c}250 \\
\text { nodes }\end{array}$ & $\begin{array}{c}300 \\
\text { nodes }\end{array}$ \\
\hline 1 & LEACH & 1.923 & 5.716 & 4.612 & 5.375 & 7.756 \\
\hline 2 & DRINA & 1.932 & 4.897 & 4.669 & 5.375 & 7.447 \\
\hline 3 & EEDRINA & 1.818 & 4.572 & 4.359 & 5.018 & 6.959 \\
\hline
\end{tabular}

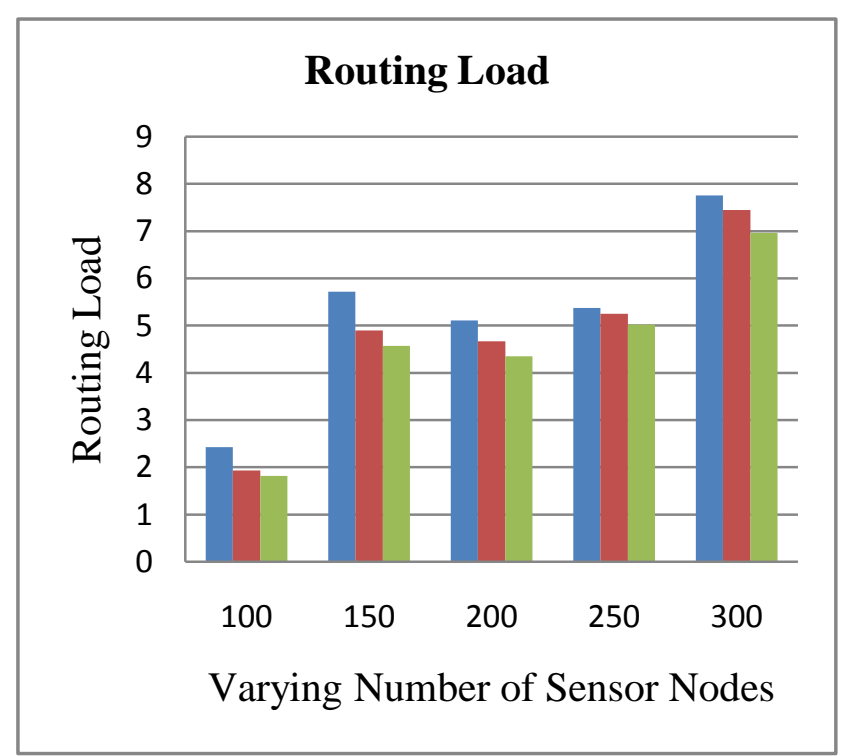

Fig. 8. Normalized Routing Load

\section{CONCLUSION}

We presented the EEDRINA algorithm, a novel and reliable Energy Efficient Data Aggregation Aware Routing Protocol for WSN. Our EEDRINA algorithm was expansively compared with two other well-known algorithms, the LEACH and DRINA, regarding energy efficiency, aggregated data delivery rate, Average delay, Routing load, Average Throughput.

EEDRINA saves nearly 10 percent Average Energy of network, improves throughput about 25 percent, minimize routing load about 5 to 10 percent also gives good results in End to End Delay and Packet Delivery Ratio. The obtain 
results clearly show that EEDRINA outperformed the LEACH and DRINA algorithms for all evaluated scenarios.

As future work, will construct the routing tree depend on application as well as consider both temporal and spatial correlation of aggregation. We will also plan to emulate modified EEDRINA algorithm on JSIM platform for greater scalability

\section{ACKNOWLEDGMENT}

I acknowledge with gratefulness and humanity my gratitude to Dr. Santosh S. Sonavane, Director of Dr. D. Y. Patil SOE Lohegaon, Pune, Maharashtra, India, for his guidance and support.

\section{REFERENCES}

[1] Wei Ye, John Heidemann, Deborah Estrin, "An energy efficient MAC protocol for wireless sensor network," IEEE INFOCOM, pp. 1567-1576, 2002

[2] Wendi Rabiner Heinzelman, Anantha Chandrakasan, HariBalakrishanan "Energy efficient communication protocol for wireless microsensor networks," IEEE 2000.

[3] Guangyu Pei, CharlrsChien, "Low power TDMA in large wireless sensor network,” IEEE pp.347-351 2001

[4] Abtin Keshavarzian, Huang Lee, Laxmi Venkatraman, "Wake up scheduling in wireless sensor networks Proc. seventh ACM Int'l Conf. Mobile Ad Hoc Networking and Computing, (pp. 322-333).2006

[5] N.A. Vasanthi and S.Annadurai., "Energy Efficient Sleep Schedule for Achieving Minimum Latency in Query Based Sensor Networks," Proc. IEEE Int'l Conf. Sensor Networks, Ubiquitous, and Trustworthy Computing, pp. 214-219, June 2006

[6] Y.Yao, J.Gehrke, "The Cougar approach to in-network query processing in sensor Networks," ACM SIGMOD 2002.

[7] Narayanan Sadagopan, BhaskarKrishnamachari, Ahmed Helmy, "The ACQUIRE mechanism for efficient ouering in sensor networks", IEEE pp. 149-155, 2003

[8] N.A.Vasanthi S.Annurai, ” AWS: Asynchronous wake up schedule to minimize latency in wireless sensor networks" ,Proc. IEEE Int'l Conf. Sensor Networks, Ubiquitous, and Trustworthy Computing, 2006

[9] Scott C.,H. Huang, Peng-Jun Wan" Minimum latency broadcast scheduling in wireless AD HOC networks" IEEE INFOCOM pp. 733-739,2007

[10] N. Bouabdallah, M.E. Rivero-Angeles, and B. Sericola, "Continuous Monitoring Using Event-Driven Reporting for Cluster-Based Wireless Sensor Networks," IEEE Trans. Vehicular Technology, vol. 58, no. 7, pp. 34603479, Sept. 2009.

[11] Yanjun Sun, Omer Gurewitz, Shu Du, Lei Tang, David Johnson, "ADB: An efficient multihop broadcast protocol based on Asynchronous duty cycling in wireless sensor networks", ACM 2009

[12] Ping Song,Xiaodong Shan, Kejie Li, Guangping Qi," Multi hop based highly precise time synchronization protocol for ZigBeenetworks" IEEE 2009.

[13] Tarek R.Sheltami, Elhadi Shakshuki," Neighbor Aware Clusterhead with Different Sleep Scheduling Protocol" IEEE2008,PP143-147
[14] A. Boukerche, B.turgut, N. Aydin, M. Z. Ahmad, L. Boloni, and D. Turgut,"Survey Paper: Routing Protocols in Ad Hoc Networks: A Survey", Computer Networks, vol.55,pp3032-3080, http://dx.doi.org/10.1.16/j.comment.2011.05.010, sept.2011

[15] J. Al-Karaki and A. Kamal,"Routing Techniques in Wireless Sensor Networks: A Survey," IEEE Wireless Comm.,vol.11,no.6,pp.6-28,Dec.2004.

[16] E. Fasolo, M. Rossi, J. Widmer, and M. Zorzi, "In Network Aggregation Techniques for Wireless Sensor Networks: A survey," IEEE Wireless Comm., vol.14,no.2,pp70-87,Apr.2007

[17] E. F.Nakamura, H.A.B.F. de Oliveira, L.F. Pontello, and A.A.F. Loureiro," On Demand Role Assignment for Event-Detection in Sensor Networks, " Proc. IEEE 11 th Symp. Computers and Comm.(ISCC '06), pp. 941947,2006

[18] A.P.Chandrakasan, A.C. Smith, and W.B. Heinzelman, "' An Application-Specific Protocol Architecture for Wireless Microsensor Networks," IEEE Trans. Wireless Comm., vol. 1, no. 4, pp. 660-670, Oct. 2002

[19] Leandro Aparecido Villas, Azzedine Boukerche, Heitor Soares Ramos, Horacio A.B., Fernandes de Oliveira, Regina Borges de Araujo, and Antonio Alfredo Ferreira Loureiro,"DRINA: A Lightweight and Reliable Routing Approach for In-Network Aggregation in Wireless Sensor Networks," IEEE Trans. On Computers, vol. 62, no. 4, pp 676-689, April 2013

[20] Xu Long-long, Zhang Jian-jun," Improved LEACH clusterhead Multi hops algorithm in wireless sensor networks" Ninth International Symposism on Distributed Computing and Applications to Business, Engineering and Science, 2010

[21] Mrs. V. Nithya, Shad drack Yaw Nusenu, Dr. B. Ramachandran" A clustering protocol basedc on tree routing algorithm in wireless sensor networks" International Conference on Advance Computing, Communication and Netorks'11 1239

[22] Bo Shen, et al," Cluster based routing protocols for wireless sensor networks" journal of software, 2006.

[23] X. H. Li, S. H. Hong, and K. L. Fang, "WSNHA-GAHR: A greedy and $\mathrm{A}^{*}$ heuristic routing algorithm for wireless sensor networks in homeautomation," IET Commun., vol. 5, no. 13, pp. 1797-1805, Sep. 2011.

[24] K.-Y. Cai and L. Zhang, "Fuzzy reasoning as a control problem," IEEE Trans. Fuzzy Syst., vol. 16, no. 3, pp. 600-614, Jun. 2008.

[25] T. A. Runkler, "Selection of appropriate defuzzification methods using application specific properties," IEEE Trans. Fuzzy Syst., vol. 5, no. 1, pp. 72-79, Feb. 1997.

[26] K. M. Passino and P. J. Antsaklis, "A metric space approach to the specification of the heuristic function for the A* algorithm," IEEE Trans.Syst. Man Cybern., vol. 24, no. 1, pp. 159-166, Jan. 1994.

[27] http://www.techopedia.com/definition/25651/wirelesssensor-network-wsn 